KYUNGPOOK Math. J. 48(2008), 1-14

\title{
Second Order Impulsive Neutral Functional Differential Inclusions
}

YICHENG LiU

Department of Mathematics and System Science, College of Science, National University of Defense Technology, Changsha, 410073, China

e-mail : liuyc2001@hotmail.com

\section{Zhixiang Li}

Department of Mathematics and System Science, College of Science, University of Defense Technology, Changsha, 410073, China

e-mail : zhxli02@yahoo.com.cn

ABSTRACT. In this paper, we investigate the existence of solutions of second order impulsive neutral functional differential inclusions which the nonlinearity $F$ admits convex and non-convex values. Some results under weaker conditions are presented. Our results extend previous ones. The methods rely on a fixed point theorem for condensing multivalued maps and Schaefer's fixed point theorem combined with lower semi-continuous multivalued operators with decomposable values.

\section{Introduction}

In this paper, we consider the existence results of solutions for the following second-order neutral functional differential inclusions with the form

$$
\begin{gathered}
{\left[p(t) u^{\prime}(t)-\int_{t-\tau}^{t} q(s) u(s) d s\right]^{\prime} \in F\left(t, u_{t}\right), \text { a.e. } t \in[0, T] \backslash\left\{t_{1}, t_{2}, \cdots, t_{m}\right\},} \\
\left.\Delta u\right|_{t=t_{k}}=I_{k}\left(u\left(t_{k}^{-}\right)\right),\left.\Delta u^{\prime}\right|_{t=t_{k}}=J_{k}\left(u\left(t_{k}^{-}\right)\right), k=1,2, \cdots, m, \\
u(t)=a(t), t \in[-\tau, 0], u^{\prime}(0)=\eta,
\end{gathered}
$$

where $F:[0, T] \times C \rightarrow \mathrm{P}\left(\mathbb{R}^{n}\right)$ is a multi-valued map, $C=\left\{\varphi:[-\tau, 0] \rightarrow \mathbb{R}^{n} ; \varphi\right.$ is continuous everywhere except for a finite number of points $\tilde{t}$ at which $\varphi\left(\tilde{t}^{-}\right)$and $\varphi\left(\tilde{t}^{+}\right)$exist with $\left.\varphi\left(\tilde{t}^{-}\right)=\varphi(\tilde{t})\right\}, a \in C, I_{k}, J_{k} \in C\left(\mathbb{R}^{n}, \mathbb{R}^{n}\right), p(\cdot) \in C\left([0, T], \mathbb{R}_{+}\right)$, $q(\cdot) \in C\left([0, T], \mathbb{R}^{n \times n}\right), \mathrm{P}\left(\mathbb{R}^{n}\right)$ is the family of all nonempty subsets of $\mathbb{R}^{n}, 0=t_{0}<$

Received June 5, 2006, and in revised form August 5, 2006.

2000 Mathematics Subject Classification: 34K45, 49K24.

Key words and phrases: Functional impulsive differential inclusions, measurable selection, condensing multi-valued map. 
$t_{1}<t_{2}<\cdots<t_{m}<t_{m+1}=T,\left.\Delta u\right|_{t=t_{k}}=u\left(t_{k}^{+}\right)-u\left(t_{k}^{-}\right), u\left(t_{k}^{+}\right)$and $u\left(t_{k}^{-}\right)$represent the right and left limits of $u(t)$ at $t=t_{k}$, respectively. $\left.\Delta u^{\prime}\right|_{t=t_{k}}$ is defined similarly. The element $u_{t} \in C$ defined by $u_{t}(\theta)=u(t+\theta)$ for $\theta \in[-\tau, 0]$.

Recently, the impulsive differential equations or inclusions were considered by Nieto[12], Cai[4], Benchohra et al.[2], Chang and Li[6] and Liu et al.[10] using various tools such as fixed point theorem, the Leray-Schauder alternative and the lower and upper solutions method. Note that when $q(s)=\mathbf{0}$, the differential inclusions (1.1)-(1.3) have been investigated by Chang and Li[6] and Benchohra, et al.[1]. In this paper, we establish the existence results for problem (1.1)-(1.3), which the nonlinearity $F$ admits convex and nonconvex values, by using the suitable fixed point theorem under weaker conditions. Our results extend the corresponding results in $[1],[2],[6]$.

The study of impulsive differential equations and inclusions is linked to their utility in simulating processes and phenomena subject to short-time perturbations during their evolution. The perturbations are performed discretely and their duration is negligible in comparison with the total duration of the processes and phenomena. That is why the perturbations are considered to take place in the form of impulses. The theory of impulsive differential equations has seen considerable development; see the monographs of Lakshmikantham et al. [8].

This paper will be divided into three sections. In Section 2 we will recall some brief basic definitions and preliminary facts which will be used in the following sections. In Sections 3 we shall establish existence theorems for (1.1)-(1.3). Our method involves reducing the existence of solutions to problem (1.1)-(1.3) to a search for fixed points of suitable multivalued maps on appropriate Banach space. In order to prove the existence of fixed points, we shall rely on a fixed point theorem for condensing multivalued maps [11] and the Schaefer's fixed point theorem [13].

\section{Preliminaries}

In this section, we list the notations, definitions and preliminary facts which are used for the rest of this paper.

By $C\left([0, T], \mathbb{R}^{n}\right)$ we denote the Banach space of all continuous functions $u$ from $[0, T]$ into $\mathbb{R}^{n}$ with the norm

$$
\|u\|_{\infty}:=\sup \{|u(t)|: t \in[0, T]\} .
$$

$L^{1}\left([0, T], \mathbb{R}^{n}\right)$ denotes the Banach space of all measurable functions $y:[0, T] \rightarrow \mathbb{R}^{n}$ which are Lebesgue integrable with the norm

$$
\|y\|_{L^{1}}:=\int_{0}^{T}|y(t)| d t
$$

Let $E$ be a nonempty subset of $\mathbb{R}^{n}$ and $N: E \rightarrow P\left(\mathbb{R}^{n}\right)$ be a multi-valued map. We say $N$ is lower semi-continuous on $E$ (in brief l.s.c.) if the set $\{x \in$ $E ; N(x) \cap C \neq \phi\}$ is open for any open set $C$ in $\mathbb{R}^{n}$. We say $N$ is upper semicontinuous on $E$ (in brief u.s.c.) if for each $x_{0} \in E$ the set $N\left(x_{0}\right)$ is a nonempty, 
closed subset of $E$, and if for each open set $C$ of $E$ containing $N\left(x_{0}\right)$, there exists an open neighborhood $B$ of $x_{0}$ such that $N(B) \subset C$. We call $N$ is closed on $E$ if its graph $G(N)=\left\{(x, y) \in E \times \mathbb{R}^{n}: y \in N(x)\right\}$ is closed. We call $N$ is compact if for each bounded set $B \subset E, N(B)$ is relatively compact. We call $N$ is bounded if for each bounded set $B \subset E, N(B)$ is bounded. We call $N$ is a condensing mapping if $\alpha(N(B))<\alpha(B)$ holds for each bounded and noncompact set $B \subset E$, where $\alpha$ denotes the noncompact measure. It is easy to see that a compact mapping must be a condensing mapping. If the multivalued map $N$ has nonempty compact values, then $N$ is u.s.c. if and only if $N$ has closed graph. The multi-valued map $N$ has a fixed point if there exists an $x \in E$ such that $x \in N(x)$.

Let $A$ denote a subset of $[0, T] \times \mathbb{R}^{n}$. We call $A$ is $L-B$ measurable if $A$ belongs to the $\sigma$-algebra generated by all sets of the form $L \times B$ where $L$ is Lebesgue measurable in $[0, T]$ and $B$ is Borel measurable in $\mathbb{R}^{n}$. A subset $S$ of $L^{1}\left([0, T], \mathbb{R}^{n}\right)$ is decomposable if for all $u, v \in S$ and all measurable subset $E$ of $[0, T]$, the function $u \chi_{E}+v_{[0, T] \backslash E} \in S$, where $\chi$ denotes the characteristic function.

For a metric space $(X, d)$, let $P_{c l}(X)=\{Y \in \mathrm{P}(X): Y$ is closed $\}, P_{c p}(X)=$ $\{Y \in \mathrm{P}(X): Y$ is compact $\}$. In order to define the solution of (1.1)-(1.3), we introduce the space $\Omega=\left\{u:[-\tau, T] \rightarrow \mathbb{R}^{n}: u \in C\left(\left(t_{k}, t_{k+1}\right), \mathbb{R}^{n}\right), u\left(t_{k}^{-}\right)\right.$and $u\left(t_{k}^{+}\right)$ exist and $u\left(t_{k}\right)=u\left(t_{k}^{-}\right), k=1, \cdots, m, u(t)=a(t)$ for $\left.t \in[-\tau, 0]\right\}$. Then $\Omega$ is a Banach space with the norm $\|u\|_{\Omega}:=\sup \{|u(t)|: t \in[-\tau, T]\}$. For a function $u$ in $\Omega$, we define $S_{F, u}$ by

$S_{F, u}=\left\{v \in L^{1}\left([-\tau, T], \mathbb{R}^{n}\right): v(t) \in F\left(t, u_{t}\right), t \in[0, T]\right.$ and $\left.v(t)=a(t), t \in[-\tau, 0]\right\}$.

Definition 2.1. A function $u \in \Omega$ is said to be a solution of (1.1)-(1.3) if $u$ satisfies the differential inclusion (1.1) a.e. on $[0, T] \backslash\left\{t_{1}, t_{2}, \cdots, t_{m}\right\}$ and the conditions (1.2)-(1.3).

Let $\Pi$ denote the set of all mappings $\varphi:[0,+\infty) \rightarrow[0,+\infty)$, where $\varphi(r)$ satisfies that there exists a strictly increasing mapping $\psi:[0,+\infty) \rightarrow[0,+\infty)$ such that $\psi(0)=0, \lim _{r \rightarrow \infty} \psi(r)=+\infty, \psi(r) \leq r-\varphi(r)$ for $r>0$.

Definition 2.2([9]). Let $(X, d)$ be a metric space and $f: X \rightarrow X$ is said to be a separate contraction mapping if there exists a function $\varphi \in \Pi$ such that $d(f(x), f(y)) \leq \varphi(d(x, y))$.

If $f: X \rightarrow X$ is a contraction mapping with Lipschitz constant $k$, then $f$ is a separate contraction mapping with $\varphi \in \Pi$ defined by $\varphi(r)=k r$. The more results on separate contraction maps we refer to the paper [9].

Definition 2.3. Let $Y$ be a separable metric space and $N: Y \rightarrow \mathrm{P}\left(L^{1}\left([0, T], \mathbb{R}^{n}\right)\right)$ be a multivalued operator. We say $N$ has property $(B C)$ if

(i) $N$ is lower semi-continuous;

(ii) $N$ has nonempty closed and decomposable values. 
The following lemmas will be used in the sequel.

Lemma 2.1([11]). Let $N: X \rightarrow P_{c p}(X)$ is a convex, u.s.c. condensing map. If the set $E=\{u \in X: u \in \lambda N(u)$, for some $\lambda \in(0,1)\}$ is bounded, then $N$ admits fixed point in $X$.

Lemma 2.2([3]). Let $Y$ be a separable metric space and $N: Y \rightarrow P\left(L^{1}\left([0, T], \mathbb{R}^{n}\right)\right)$ be a multivalued map with the property $(B C)$. Then $N$ has a continuous selection, i.e., there exists a continuous function (single-value) $g: Y \rightarrow L^{1}\left([0, T], \mathbb{R}^{n}\right)$ such that $g(u) \in N(u)$ for every $u \in Y$.

Lemma 2.3([13]). Let $(X,\|\cdot\|)$ be a normed space. $H$ is a continuous mapping of $X$ into $X$ which is compact on each bounded subset of $X$. Then either

(i) $x=\lambda H x$ has a solution in $X$ for $\lambda=1$, or

(ii) the set of all such solutions, $0<\lambda<1$, is unbounded.

\section{Existence results}

In this section, we shall prove our main results. Firstly, due to the fixed point theorem of Martelli [11] for condensing multi-valued map, we establish the existence result for the problem (1.1)-(1.3) which the nonlinearity $F$ is restricted to be convexvalued. Let $p_{0}=\min _{t \in[0, T]}|p(t)|, \tilde{p}=\max _{t \in[0, T]}|p(t)|$ and

$$
\mu=p(0) \eta-\int_{-\tau}^{0} q(s) a(s) d s, \quad q=\sup _{t \in[0, T]} \int_{t-\tau}^{t}|q(s)| d s .
$$

Now we give the following assumptions.

$\left(\mathrm{H}_{1}\right) F(\cdot, u):[0, T] \rightarrow P_{c p}\left(\mathbb{R}^{n}\right)$ is convex and measurable for each $u \in C$ and satisfies $u \rightarrow F(t, u)$ is u.s.c. for a.e. $t \in[0, T]$.

$\left(\mathrm{H}_{2}\right)$ There exist two constants $c_{k}, c_{k}^{\prime}$ such that $\left|I_{k}(x)\right| \leq c_{k}, \quad\left|J_{k}(x)\right| \leq c_{k}^{\prime}$, for each $k=1, \cdots, m$ and all $x \in \mathbb{R}^{n}$.

$\left(\mathrm{H}_{3}\right)\|F(t, u)\|=\sup \{|v(t)|: v(t) \in F(t, u)\} \leq l(t) \chi(\|u\|)$ for a.e. $t \in[0, T]$ and all $u \in C$, where $l \in L^{1}\left([0, T], \mathbb{R}_{+}\right)$and $\chi: \mathbb{R}_{+} \rightarrow(0,+\infty)$ is continuous and increasing with

$$
\int_{0}^{T}\left[p^{-1}(t)+p(t) l(t) \int_{t}^{T} p^{-2}(r) d r\right] d t<\int_{c}^{\infty} \frac{d v}{\chi(v)+q v},
$$

where $c=|a(0)|+T p_{0}^{-1}|\mu|+\sum_{i=1}^{m}\left[c_{k}+\frac{\left(T-t_{k}\right) p\left(t_{k}\right)}{p_{0}} c_{k}^{\prime}\right]$. 
Remark 3.1. In the case $p(t) \equiv 1$ and $q(s) \equiv 0$, the assumption $\left(H_{3}\right)$ has been considered by some authors $[1,2]$. Based on $\left(H_{1}\right)$ and $\left(H_{3}\right)$, we see that $F$ is an $L^{1}$ Carathéodory multivalued map.

Theorem 3.1. Assume that $\left(H_{1}\right)-\left(H_{3}\right)$ hold. Then the problem $(1.1)-(1.3)$ has at least one solution on $[-\tau, T]$.

Proof. We transform the problem (1.1)-(1.3) into a fixed point problem. Consider the multi-valued map $M: \Omega \rightarrow \mathrm{P}(\Omega)$, defined by $M(u)=\{h \in \Omega\}$, where

$$
\begin{aligned}
h(t)= & a(0)+\int_{0}^{t} p^{-1}(r) \int_{r-\tau}^{r} q(s) u(s) d s d r+\int_{0}^{t} \int_{s}^{t} p^{-2}(r) d r p(s) g(s) d s \\
& +\int_{0}^{t} p^{-1}(r) d r \mu+\sum_{0<t_{k}<t}\left[I_{k}\left(u\left(t_{k}^{-}\right)\right)+\int_{t_{k}}^{t} \frac{p\left(t_{k}\right)}{p(r)} d r J_{k}\left(u\left(t_{k}^{-}\right)\right)\right] \\
& , t \in[0, T], g \in S_{F, u}
\end{aligned}
$$

and $h(t)=a(t)$ for $t \in[-\tau, 0]$. It is clear that the fixed points of $M$ are solutions to the problem (1.1)-(1.3). For each $u \in \Omega$ the set $S_{F, u}$ is nonempty since, by $\left(H_{1}\right)$, $F$ has a measurable selection. We shall prove that $M$ fulfills the assumptions of Lemma 2.1. The proof will be given by several steps.

Step 1. $M(u)$ is convex for each $u \in \Omega$. In fact, if $h_{1}, h_{2}$ belong to $M(u)$, then there exist $g_{1}, g_{2} \in S_{F, u}$ such that for each $t \in[0, T]$

$$
\begin{aligned}
h_{i}(t)= & a(0)+\int_{0}^{t} p^{-1}(r) \int_{r-\tau}^{r} q(s) u(s) d s d r+\int_{0}^{t} \int_{s}^{t} p^{-2}(r) d r p(s) g_{i}(s) d s \\
& +\int_{0}^{t} p^{-1}(r) d r \mu+\sum_{0<t_{k}<t}\left[I_{k}\left(u\left(t_{k}^{-}\right)\right)+\int_{t_{k}}^{t} \frac{p\left(t_{k}\right)}{p(r)} d r J_{k}\left(u\left(t_{k}^{-}\right)\right)\right], i=1,2 .
\end{aligned}
$$

For any $d \in[0,1]$, we have

$$
\begin{aligned}
& \left(d h_{1}+(1-d) h_{2}\right)(t) \\
= & a(0)+\int_{0}^{t} p^{-1}(r) \int_{r-\tau}^{r} q(s) u(s) d s d r+\int_{0}^{t} \int_{s}^{t} p^{-2}(r) d r p(s)\left(d g_{1}+(1-d) g_{2}\right)(s) d s \\
& +\int_{0}^{t} p^{-1}(r) d r \mu+\sum_{0<t_{k}<t}\left[I_{k}\left(u\left(t_{k}^{-}\right)\right)+\int_{t_{k}}^{t} \frac{p\left(t_{k}\right)}{p(r)} d r J_{k}\left(u\left(t_{k}^{-}\right)\right)\right] .
\end{aligned}
$$

Since $S_{F, u}$ is convex (because $F$ has convex values) then $d h_{1}+(1-d) h_{2} \in M(u)$.

Step 2. $M$ maps bounded sets into relatively compact sets in $\Omega$.

Let $B_{r}=\left\{u \in \Omega:\|u\|_{\Omega} \leq r\right\}$ be a bounded set in $\Omega$ and $u \in B_{r}$, we obtain that, based upon the assumptions $\left(H_{2}\right)$ and $\left(H_{3}\right), M\left(B_{r}\right)$ is bounded. 
On the other hand, let $d=\max \left\{\left|c_{k}^{\prime}\right|: k=1, \cdots, m\right\}$. Then, for $\tau_{1}, \tau_{2} \in$ $\left[t_{k-1}, t_{k}\right], k=1,2, \cdots, m$ and $u \in B_{r}$, we have

$$
\begin{aligned}
& \left|M(u)\left(\tau_{2}\right)-M(u)\left(\tau_{1}\right)\right| \\
\leq & \left|\int_{\tau_{1}}^{\tau_{2}} p^{-1}(r) \int_{r-\tau}^{r} q(s) u(s) d s d r\right|+\left|\int_{\tau_{1}}^{\tau_{2}} \int_{s}^{\tau_{2}} p^{-2}(r) d r p(s) g(s) d s\right| \\
& +\left|\int_{0}^{\tau_{1}} \int_{\tau_{1}}^{\tau_{2}} p^{-2}(r) d r p(s) g(s) d s\right|+\left|\int_{\tau_{1}}^{\tau_{2}} p^{-1}(r) d r \mu\right|+\left|\sum_{\tau_{1}<t_{k}<\tau_{2}} I_{k}\left(u\left(t_{k}^{-}\right)\right)\right| \\
& +\frac{\tilde{p}}{p_{0}} d\left|\tau_{2}-\tau_{1}\right| .
\end{aligned}
$$

As $\left|\tau_{2}-\tau_{1}\right| \rightarrow 0$, the right hand side of the above inequality tends to zero. The case $\tau_{1}, \tau_{2} \in[-\tau, 0]$ is obvious. Thus for any sequence $\left\{h_{n}\right\} \subset M\left(B_{r}\right)$. By above arguments and Ascoli-Arzela theorem, we could choose a common subsequence $\left\{h_{n_{k}}\right\}$ such that $h_{n_{k}}(t)$ uniformly converges to $h_{0}^{j}(t)$ as $k$ tending to infinity for $t \in\left[t_{j}, t_{j+1}\right]$, $j=0,1,2, \cdots, m$. Take

$$
h_{0}(t)=\left\{\begin{array}{l}
a(t), t \in[-\tau, 0] \\
h_{0}^{j}(t), t \in\left(t_{j-1}, t_{j}\right], j=1,2, \cdots, m+1,
\end{array}\right.
$$

then $h_{0} \in \Omega$. Thus the subsequence $h_{n_{k}}(t)$ uniformly converges to $h_{0}(t)$ on $[-\tau, T]$. This implies that $M\left(B_{r}\right)$ is a relatively compact set.

Step 3. The set $E=\{u \in \Omega: u \in \lambda M(u)$, for some $\lambda \in(0,1)\}$ is bounded.

Let $u \in E$, that is, $u \in \lambda M(u)$ for some $\lambda \in(0,1)$. Thus there exists $g \in S_{F, u}$ such that

$$
\begin{aligned}
u(t)= & \lambda a(0)+\lambda \int_{0}^{t} p^{-1}(r) \int_{r-\tau}^{r} q(s) u(s) d s d r+\lambda \int_{0}^{t} \int_{s}^{t} p^{-2}(r) d r p(s) g(s) d s \\
& +\lambda \int_{0}^{t} p^{-1}(r) d r \mu+\lambda \sum_{0<t_{k}<t}\left[I_{k}\left(u\left(t_{k}^{-}\right)\right)+\int_{t_{k}}^{t} \frac{p\left(t_{k}\right)}{p(r)} d r J_{k}\left(u\left(t_{k}^{-}\right)\right)\right] .
\end{aligned}
$$

By assumptions $\left(H_{2}\right)$ and $\left(H_{3}\right)$, we achieve that

$$
\begin{aligned}
|u(t)| \leq & |a(0)|+\int_{0}^{t} p^{-1}(r) \int_{r-\tau}^{r}|q(s) \| u(s)| d s d r+T p_{0}^{-1}|\mu| \\
& +\int_{0}^{t} \int_{s}^{T} p^{-2}(r) \operatorname{drp}(s) l(s) \chi\left(\left\|u_{s}\right\|\right) d s+\sum_{i=1}^{m}\left[c_{k}+\frac{\left(T-t_{k}\right) p\left(t_{k}\right)}{p_{0}} c_{k}^{\prime}\right] .
\end{aligned}
$$

Let

$$
\zeta(t)=\sup \{|u(s)|:-\tau \leq s \leq t\}, t \in[0, T]
$$


Then we have

$$
\begin{aligned}
\zeta(t) \leq & |a(0)|+\int_{0}^{t} p^{-1}(r) \zeta(r) \int_{r-\tau}^{r}|q(s)| d s d r+T p_{0}^{-1}|\mu| \\
& +\int_{0}^{t} \int_{s}^{T} p^{-2}(r) d r p(s) l(s) \chi(\zeta(s)) d s+\sum_{i=1}^{m}\left[c_{k}+\frac{\left(T-t_{k}\right) p\left(t_{k}\right)}{p_{0}} c_{k}^{\prime}\right] .
\end{aligned}
$$

Take the right-hand side of the above inequality as $v(t)$, then we obtain $\zeta(t) \leq v(t)$ and

$$
v(0)=|a(0)|+T p_{0}^{-1}|\mu|+\sum_{i=1}^{m}\left[c_{k}+\frac{\left(T-t_{k}\right) p\left(t_{k}\right)}{p_{0}} c_{k}^{\prime}\right]=c .
$$

Thus

$$
\begin{aligned}
v^{\prime}(t) & \leq p^{-1}(t) q v(t)+\int_{t}^{T} p^{-2}(r) d r p(t) l(t) \chi(v(t)) \\
& \leq\left[p^{-1}(t)+p(t) l(t) \int_{t}^{T} p^{-2}(r) d r\right][\chi(v(t))+q v(t)]
\end{aligned}
$$

This implies that

$$
\int_{v(0)}^{v(t)} \frac{d v}{\chi(v)+q v} \leq \int_{0}^{T}\left[p^{-1}(t)+p(t) l(t) \int_{t}^{T} p^{-2}(r) d r\right] d t<\int_{v(0)}^{\infty} \frac{d v}{\chi(v)+q v} .
$$

Hence, there exists a constant $Q$ such that $v(t) \leq Q$ for $t \in[-\tau, T]$. Thus $\|u\|_{\Omega} \leq Q$. This proves that the set $E$ is bounded.

Following similar arguments with [1, p.376-377], we conclude that $M$ has closed graph. So the multi-valued mapping $M$ is u.s.c.. Meanwhile, the compact map $M$ is a condensing map. Thus $M$ fulfills the all assumptions of Lemma 2.1, then $M$ admits a fixed point which is a solution to the problem (1.1)-(1.3). The proof of Theorem 3.1 is complete.

Based on the Schaefer's theorem combined with the selection theorem of Bressan and Colombo for semi-continuous maps with decomposable values, we will establish some new existence results for problem (1.1)-(1.3) which the nonlinearity $F$ admits nonconvex-valued. We consider the following assumptions.

$\left(\mathrm{H}_{4}\right)$ Assume that $F:[0, T] \times C \rightarrow P_{c p}\left(\mathbb{R}^{n}\right)$ satisfy

(1) $(t, u) \rightarrow F(t, u)$ is $L-B$ measurable;

(2) $u \rightarrow F(t, u)$ is l.s.c. for a.e. $t \in[0, T]$.

$\left(\mathrm{H}_{5}\right)$ There exists a function $D \in L^{1}\left([0, T], \mathbb{R}_{+}\right)$such that, for a.e. $t \in[0, T]$ and $u \in C$,

$$
\|F(t, u)\|=\sup \{|v(t)|: v(t) \in F(t, u)\} \leq D(t) .
$$


$\left(\mathrm{H}_{6}\right)$ There exists a function $\varphi \in \Pi$ and a constant $b>0$ such that

$$
\|\Phi(u)\|_{\Omega} \leq \varphi\left(\|u\|_{\Omega}\right)+b
$$

where $u \in \Omega$ and $\Phi: \Omega \rightarrow \Omega$ defined by

$$
\begin{aligned}
& \Phi(u)(t) \\
= & \sum_{0<t_{k}<t}\left[I_{k}\left(u\left(t_{k}^{-}\right)\right)+\int_{t_{k}}^{t} \frac{p\left(t_{k}\right)}{p(r)} d r J_{k}\left(u\left(t_{k}^{-}\right)\right)\right]+\int_{0}^{t} p^{-1}(r) \int_{r-\tau}^{r} q(s) u(s) d s d r .
\end{aligned}
$$

Lemma 3.1([7]). Let $F:[0, T] \times C \rightarrow P_{c p}\left(\mathbb{R}^{n}\right)$ be a multi-valued map and the assumptions $\left(H_{4}\right)$ and $\left(H_{5}\right)$ hold. Then $F$ is lower semicontinuous type.

We say $F$ is lower semicontinuous type if its Niemytzki operator

$$
\mathrm{F}(u):=\left\{w \in L^{1}\left([0, T], \mathbb{R}^{n}\right): w(t) \in F\left(t, u_{t}\right) \text { for a.e. } t \in[0, T]\right\}
$$

is lower semicontinuous and nonempty closed and decomposable values.

Theorem 3.2. Assume that $\left(H_{4}\right)-\left(H_{6}\right)$ hold. Then the problem $(1.1)-(1.3)$ admits at least one solution on $[-\tau, T]$.

Proof. Note that $\left(H_{4}\right)$ and $\left(H_{5}\right)$ and Lemma 3.1 imply that $F$ is lower semicontinuous type. By Lemma 2.2, there exists a continuous function $f: \Omega \rightarrow L^{1}\left([0, T], \mathbb{R}^{n}\right)$ such that $f(u) \in \mathrm{F}(u)$ for all $u \in \Omega$.

We consider the corresponding problem

$$
\begin{gathered}
{\left[p(t) u^{\prime}(t)-\int_{t-\tau}^{t} q(s) u(s) d s\right]^{\prime}=f\left(u_{t}\right), \text { a.e. } t \in[0, T] \backslash\left\{t_{1}, t_{2}, \cdots, t_{m}\right\}} \\
\left.\Delta u\right|_{t=t_{k}}=I_{k}\left(u\left(t_{k}^{-}\right)\right),\left.\Delta u^{\prime}\right|_{t=t_{k}}=J_{k}\left(u\left(t_{k}^{-}\right)\right), k=1,2, \cdots, m, \\
u(t)=a(t), t \in[-\tau, 0], u^{\prime}(0)=\eta .
\end{gathered}
$$

It is clear that if $u \in \Omega$ is a solution of (3.1)-(3.3), then $u$ is a solution to problem (1.1)-(1.3). Transform the problem (3.1)-(3.3) into a fixed point problem. Consider the operators $\Lambda: \Omega \rightarrow \Omega$ defined by

$$
\begin{aligned}
\Lambda(u)(t) & =a(0)+\int_{0}^{t} p^{-1}(r) \int_{r-\tau}^{r} q(s) u(s) d s d r+\int_{0}^{t} p^{-1}(s) \int_{0}^{s} f\left(u_{r}\right) d r d s \\
& +\int_{0}^{t} p^{-1}(r) d r \mu+\sum_{0<t_{k}<t}\left[I_{k}\left(u\left(t_{k}^{-}\right)\right)+\int_{t_{k}}^{t} \frac{p\left(t_{k}\right)}{p(r)} d r J_{k}\left(u\left(t_{k}^{-}\right)\right)\right]
\end{aligned}
$$


for $t \in[0, T]$ and $\Lambda(u)(t)=a(t)$ for $t \in[-\tau, 0]$. We shall prove that $\Lambda$ fulfills the assumptions of Lemma 2.3. It is clear that $\Lambda$ is continuous by the continuities of functions $f, I_{k}$ and $J_{k}$. The rest of proof will be given by following two steps.

Step 1. $\Lambda$ maps bounded sets into relatively compact sets in $\Omega$.

Let $B_{r}=\left\{u \in \Omega:\|u\|_{\Omega} \leq r\right\}$ be a bounded set in $\Omega$ and $u \in B_{r}$, we obtain, based upon the assumptions $\left(H_{5}\right)$ and $\left(H_{6}\right)$,

$$
\begin{aligned}
& |\Lambda(u)(t)| \\
\leq & |a(0)|+p_{0}^{-1} T|\mu|+p_{0}^{-1} T \int_{0}^{T} D(s) d s \\
& +\left|\sum_{0<t_{k}<t}\left[I_{k}\left(u\left(t_{k}^{-}\right)\right)+\int_{t_{k}}^{t} \frac{p\left(t_{k}\right)}{p(r)} d r J_{k}\left(u\left(t_{k}^{-}\right)\right)\right]+\int_{0}^{t} p^{-1}(r) \int_{r-\tau}^{r} q(s) u(s) d s d r\right| \\
\leq & |a(0)|+p_{0}^{-1} T|\mu|+\varphi\left(\|u\|_{\Omega}\right)+b \\
\leq & |a(0)|+p_{0}^{-1} T|\mu|+r+b:=M .
\end{aligned}
$$

Thus, for each $u \in B_{r}$, we have $\|\Lambda(u)\|_{\Omega} \leq M$.

On the other hand, let $J_{r}=\max \left\{\left|J_{k}(x)\right|:|x| \leq r, k=1, \cdots, m\right\}$. Then, for $\tau_{1}, \tau_{2} \in\left[t_{k-1}, t_{k}\right], k=1,2, \cdots, m$ and $u \in B_{r}$, we have

$$
\begin{aligned}
& \left|\Lambda(u)\left(\tau_{2}\right)-\Lambda(u)\left(\tau_{1}\right)\right| \\
\leq & \left|\int_{\tau_{1}}^{\tau_{2}} p^{-1}(r) \int_{r-\tau}^{r} q(s) u(s) d s d r\right|+\left|\int_{\tau_{1}}^{\tau_{2}} p^{-1}(s) \int_{0}^{s} f\left(u_{r}\right) d r d s\right| \\
& +\left|\int_{\tau_{1}}^{\tau_{2}} p^{-1}(r) d r \mu\right|+\left|\sum_{\tau_{1}<t_{k}<\tau_{2}} I_{k}\left(u\left(t_{k}^{-}\right)\right)\right|+\frac{\tilde{p}}{p_{0}} J_{r}\left|\tau_{2}-\tau_{1}\right| .
\end{aligned}
$$

As $\left|\tau_{2}-\tau_{1}\right| \rightarrow 0$, the right hand side of the above inequality tends to zero. The case $\tau_{1}, \tau_{2} \in[-\tau, 0]$ is similar. Thus for any sequence $\left\{h_{n}\right\} \subset \Lambda\left(B_{r}\right)$. By above arguments and Ascoli-Arzela theorem, we can choose a common subsequence $\left\{h_{n_{k}}\right\}$ such that $h_{n_{k}}(t)$ uniformly converges to $h_{0}^{j}(t)$ as $k$ tending to infinity for $t \in\left[t_{j}, t_{j+1}\right]$, $j=0,1,2, \cdots, m$. Take

$$
h_{0}(t)= \begin{cases}a(t), & t \in[-\tau, 0] \\ h_{0}^{j}(t), & t \in\left(t_{j-1}, t_{j}\right], j=1,2, \cdots, m+1\end{cases}
$$

then $h_{0} \in \Omega$. Thus the subsequence $h_{n_{k}}(t)$ uniformly converges to $h_{0}(t)$ on $[-\tau, T]$. This implies that $\Lambda\left(B_{r}\right)$ is a relatively compact set.

Step 2. The set $E=\{u \in \Omega: \exists \theta \in(0,1), u=\theta \Lambda(u)\}$ is bounded.

Indeed, let $u \in E$, then $u(t)=\theta \Lambda(u)(t)$ for some $\theta \in(0,1)$. Note that $\left(H_{6}\right)$, we obtain

$$
\begin{aligned}
|u(t)| & \leq|a(0)|+p_{0}^{-1} T\|D\|_{L^{1}}+p_{0}^{-1} T|\mu|+\theta \varphi\left(\|u\|_{\Omega}\right)+\theta b \\
& \leq s+\varphi\left(\|u\|_{\Omega}\right)
\end{aligned}
$$


where $s=|\varphi(0)|+p_{0}^{-1} T\|D\|_{L^{1}}+p_{0}^{-1} T|\mu|+b$. Thus we have $\|u\|_{\Omega} \leq s+\varphi\left(\|u\|_{\Omega}\right)$. Since $\varphi(\cdot) \in \Pi$, we can achieve

$$
\psi\left(\|u\|_{\Omega}\right) \leq\|u\|_{\Omega}-\varphi\left(\|u\|_{\Omega}\right) \leq s .
$$

Hence, from the monotonousness of $\psi(\cdot)$, we get $\|u\|_{\Omega} \leq \psi^{-1}(s)<\infty$. This implies that the set $E$ is bounded.

In view of Lemma 2.3 , we deduce that $\Lambda$ has a fixed point which is a solution of the problem (1.1)-(1.3).

Corollary 3.1. Assume that $\left(H_{4}\right)$ and $\left(H_{5}\right)$ holds. In addition, if one of following conditions hold :

$\left(\mathrm{A}_{1}\right)$ there exists $\varphi \in \Pi$ such that $\|\Phi(x)-\Phi(y)\|_{\Omega} \leq \varphi\left(\|x-y\|_{\Omega}\right)$.

$\left(\mathrm{A}_{2}\right)$ there exist constants $c_{k}, c_{k}^{\prime}, d_{k}$ and $b_{k}$ such that, for each $x \in \mathbb{R}^{n}$,

$$
\left|I_{k}(x)\right| \leq c_{k}|x|+b_{k},\left|J_{k}(x)\right| \leq c_{k}^{\prime}|x|+d_{k}, \sum_{k=1}^{m}\left(c_{k}+\frac{T-t_{k}}{p_{0}} p\left(t_{k}\right) c_{k}^{\prime}\right)<1-\frac{T q}{p_{0}} .
$$

Then the problem $(1.1)-(1.3)$ admits at least one solution on $[-\tau, T]$.

Proof. We only show the conditions $\left(A_{1}\right)$ and $\left(A_{2}\right)$ imply the assumption $\left(H_{6}\right)$ respectively. First, if the condition $\left(A_{1}\right)$ holds, then there exists a function $\varphi \in \Pi$ such that

$$
\|\Phi(u)-\Phi(v)\|_{\Omega} \leq \varphi\left(\|u-v\|_{\Omega}\right),
$$

where $u, v \in \Omega$. Take $v=0$ and $b:=\sum_{k=1}^{m}\left(\left|I_{k}(0)\right|+\frac{T-t_{k}}{p_{0}} p\left(t_{k}\right)\left|J_{k}(0)\right|\right) \geq$ $\|\Phi(0)\|_{\Omega}$, we obtain the assumption $\left(H_{6}\right)$ immediately.

If the condition $\left(A_{2}\right)$ holds, for each $t \in[0, T]$, we have

$$
\begin{aligned}
|\Phi(u)(t)|= & \left|\sum_{0<t_{k}<t}\left[I_{k}\left(u\left(t_{k}^{-}\right)\right)+\int_{t_{k}}^{t} \frac{p\left(t_{k}\right)}{p(r)} d r J_{k}\left(u\left(t_{k}^{-}\right)\right)\right]\right|+p_{0}^{-1} T q\|u\|_{\Omega} \\
\leq & \sum_{k=1}^{m}\left(c_{k}\left|u\left(t_{k}^{-}\right)\right|+\frac{T-t_{k}}{p_{0}} p\left(t_{k}\right) c_{k}^{\prime}\left|u\left(t_{k}^{-}\right)\right|\right) \\
& +\sum_{k=1}^{m}\left(b_{k}+\frac{T-t_{k}}{p_{0}} p\left(t_{k}\right) d_{k}\right)+p_{0}^{-1} T q\|u\|_{\Omega} \\
\leq & {\left[\sum_{k=1}^{m}\left(c_{k}+\frac{T-t_{k}}{p_{0}} p\left(t_{k}\right) c_{k}^{\prime}\right)+p_{0}^{-1} T q\right]\|u\|_{\Omega}+\sum_{k=1}^{m}\left(b_{k}+\frac{T-t_{k}}{p_{0}} p\left(t_{k}\right) d_{k}\right) . }
\end{aligned}
$$

Thus

$$
\|\Phi(u)\|_{\Omega} \leq\left[\sum_{k=1}^{m}\left(c_{k}+\frac{T-t_{k}}{p_{0}} p\left(t_{k}\right) c_{k}^{\prime}\right)+p_{0}^{-1} T q\right]\|u\|_{\Omega}+\sum_{k=1}^{m}\left(b_{k}+\frac{T-t_{k}}{p_{0}} p\left(t_{k}\right) d_{k}\right) .
$$


By $\sum_{k=1}^{m}\left(c_{k}+\frac{T-t_{k}}{p_{0}} p\left(t_{k}\right) c_{k}^{\prime}\right)<1-\frac{T q}{p_{0}}$, we define a function as $\varphi(r)=$ $\left[\sum_{k=1}^{m}\left(c_{k}+\frac{T-t_{k}}{p_{0}} p\left(t_{k}\right) c_{k}^{\prime}\right)+p_{0}^{-1} T q\right] r$ for $r>0$. Then $\varphi \in \Pi$ fulfills the assumption $\left(H_{6}\right)$. Thus it follows from Theorem 3.2 that the problem (1.1)-(1.3) admits a solution in $\Omega$. The proof of Corollary 3.1 is complete.

From the above proof of Theorem 3.2, we immediately obtain the following corollaries.

Corollary 3.2. Assume that $\left(H_{4}\right)$ and $\left(H_{5}\right)$ hold. Then the problem $(1.1)-(1.3)$ admits at least one solution on $[-\tau, T]$, provided

$$
\left|I_{k}(x)-I_{k}(y)\right| \leq c_{k}|x-y|,\left|J_{k}(x)-J_{k}(y)\right| \leq c_{k}^{\prime}|x-y|, k=1,2, \cdots, m
$$

and

$$
\sum_{k=1}^{m}\left(c_{k}+\frac{T-t_{k}}{p_{0}} p\left(t_{k}\right) c_{k}^{\prime}\right)<1-\frac{T q}{p_{0}}
$$

Corollary 3.3. Assume that $\left(H_{2}\right),\left(H_{4}\right)$ and $\left(H_{5}\right)$ hold. Then the problem (1.1)(1.3) admits at least one solution on $[-\tau, T]$, provided $T q<p_{0}$.

Theorem 3.3. Assume that $\left(H_{4}\right),\left(H_{5}\right)$ and the following condition hold:

$\left(\mathrm{H}_{7}\right) \quad 0 \leq \varlimsup_{|x| \rightarrow \infty} \frac{\left|I_{k}(x)\right|}{|x|} \leq c_{k}, k=1, \cdots, m ;$

$\left(\mathrm{H}_{8}\right) \quad 0 \leq \varlimsup_{|x| \rightarrow \infty} \frac{\left|J_{k}(x)\right|}{|x|} \leq c_{k}^{\prime}, k=1, \cdots, m$.

Then the problem $(1.1)-(1.3)$ admits at least one solution on $[-\tau, T]$, provided

$$
\sum_{k=1}^{m}\left(c_{k}+\frac{T-t_{k}}{p_{0}} p\left(t_{k}\right) c_{k}^{\prime}\right)<1-\frac{T q}{p_{0}} .
$$

Proof. Firstly, we transform the problem (3.1)-(3.3) into a fixed point problem. Consider the operators $A: \Omega \rightarrow \Omega$ defined by

$$
\begin{aligned}
A(u)(t)= & a(0)+\int_{0}^{t} p^{-1}(r) \int_{r-\tau}^{r} q(s) u(s) d s d r+\int_{0}^{t} p^{-1}(s) \int_{0}^{s} f\left(u_{r}\right) d r d \\
+ & \int_{0}^{t} p^{-1}(r) d r\left[p(0) \eta-\int_{-\tau}^{0} q(s) a(s) d s\right] \\
& +\sum_{0<t_{k}<t}\left[I_{k}\left(u\left(t_{k}\right)\right)+\int_{t_{k}}^{t} \frac{p\left(t_{k}\right)}{p(r)} d r J_{k}\left(u\left(t_{k}\right)\right)\right]
\end{aligned}
$$


for $t \in[0, T]$ and $A(u)(t)=a(t)$ for $t \in[-\tau, 0]$. We shall prove that $A$ fulfills the assumptions of Lemma 2.3. Based on the step 1 in proof of Theorem 3.2, we only prove the set $E=\{u \in \Omega: \exists \lambda \in(0,1), u=\lambda A(u)\}$ is bounded. From conditions $\left(H_{7}\right)$ and $\left(H_{8}\right)$, we conclude that there exist positive constants $\varepsilon_{k}, \varepsilon_{k}^{\prime}, k=1, \cdots, m$ and $K$ such that, for all $|u|>K$,

$$
\left|I_{k}(u)\right| \leq\left(c_{k}+\varepsilon_{k}\right)|u|,\left|J_{k}(u)\right| \leq\left(c_{k}^{\prime}+\varepsilon_{k}^{\prime}\right)|u|,
$$

and

$$
\sum_{k=1}^{m}\left(c_{k}+\varepsilon_{k}+\frac{T-t_{k}}{p_{0}} p\left(t_{k}\right)\left(c_{k}^{\prime}+\varepsilon_{k}^{\prime}\right)\right)<1-\frac{T q}{p_{0}}
$$

We prove $E$ is bounded by contradiction. Suppose that there exists a sequence $u_{n} \in E$ such that $\left\|u_{n}\right\|_{\Omega} \rightarrow+\infty$ as $n \rightarrow+\infty$. For any constant $C_{1}>K$ and each $n$, we define

$$
\begin{aligned}
D_{n} & =\left\{t: t \in[0, T],\left|u_{n}(t)\right| \leq C_{1}\right\} ; \quad L=\max \left\{\left|I_{k}(x)\right|:|x| \leq C_{1}\right\} ; \\
L^{\prime} & =\max \left\{\left|J_{k}(x)\right|:|x| \leq C_{1}\right\} ; \quad L_{n}=\max \left\{\left|I_{k}\left(u_{n}(t)\right)\right|: t \in D_{n}\right\} ; \\
L_{n}^{\prime} & =\max \left\{\left|J_{k}\left(u_{n}(t)\right)\right|: t \in D_{n}\right\} .
\end{aligned}
$$

It is clear that $L_{n} \leq L$ and $L_{n}^{\prime} \leq L^{\prime}$ for each $n$. Then, for each $n$, we have

$$
\begin{aligned}
\left|u_{n}(t)\right| \leq & |a(0)|+\left|\int_{0}^{T} p^{-1}(r) \int_{r-\tau}^{r} q(s) u(s) d s d r\right|+\left|\int_{0}^{t} p^{-1}(s) \int_{0}^{s} f\left(u_{r}\right) d r d s\right| \\
& +\left|\int_{0}^{T} p^{-1}(r) d r \mu\right|+\left|\sum_{0<t_{k}<t}\left[I_{k}\left(u_{n}\left(t_{k}\right)\right)+\int_{t_{k}}^{t} \frac{p\left(t_{k}\right)}{p(r)} d r J_{k}\left(u_{n}\left(t_{k}\right)\right)\right]\right| \\
\leq & S+\frac{T q}{p_{0}}\left\|u_{n}\right\|_{\Omega}+\sum_{t_{k} \in D_{n}}\left|I_{k}\left(u_{n}\left(t_{k}^{-}\right)\right)\right|+\sum_{t_{k} \in[0, T] \backslash D_{n}}\left|I_{k}\left(u_{n}\left(t_{k}^{-}\right)\right)\right| \\
& +\frac{T \tilde{p}}{p_{0}} \sum_{t_{k} \in D_{n}}\left|J_{k}\left(u_{n}\left(t_{k}^{-}\right)\right)\right|+\sum_{t_{k} \in[0, T] \backslash D_{n}} \frac{T-t_{k}}{p_{0}} p\left(t_{k}\right)\left|J_{k}\left(u_{n}\left(t_{k}^{-}\right)\right)\right| \\
\leq & S+m L+\frac{T \tilde{p}}{p_{0}} m L^{\prime}+\sum_{t_{k} \in[0, T] \backslash D_{n}}\left|I_{k}\left(u_{n}\left(t_{k}^{-}\right)\right)\right| \\
& +\frac{T-t_{k}}{p_{0}} p\left(t_{k}\right) \sum_{t_{k} \in[0, T] \backslash D_{n}}\left|J_{k}\left(u_{n}\left(t_{k}^{-}\right)\right)\right|+\frac{T q}{p_{0}}\left\|u_{n}\right\|_{\Omega} \\
\leq & S+m L+\frac{T \tilde{p}}{p_{0}} m L^{\prime}+\left[\sum_{k=1}^{m}\left(c_{k}+\varepsilon_{k}+\frac{T-t_{k}}{p_{0}} p\left(t_{k}\right)\left(c_{k}^{\prime}+\varepsilon_{k}^{\prime}\right)\right)+\frac{T q}{p_{0}}\right]\left\|u_{n}\right\|_{\Omega},
\end{aligned}
$$

where $S=|a(0)|+\left|\int_{0}^{T} p^{-1}(r) d r\left[p(0) \eta-\int_{-\tau}^{0} q(s) a(s) d s\right]\right|+p_{0}^{-1} T\|D\|_{L^{1}}$. Thus we 
have

$\left\|u_{n}\right\|_{\Omega} \leq S+m L+\frac{T \tilde{p}}{p_{0}} m L^{\prime}+\left[\sum_{k=1}^{m}\left(c_{k}+\varepsilon_{k}+\frac{T-t_{k}}{p_{0}} p\left(t_{k}\right)\left(c_{k}^{\prime}+\varepsilon_{k}^{\prime}\right)\right)+\frac{T q}{p_{0}}\right]\left\|u_{n}\right\|_{\Omega}$.

This implies

$$
\left\|u_{n}\right\|_{\Omega} \leq \frac{S+m L+\frac{T \tilde{p}}{p_{0}} m L^{\prime}}{1-\left[\sum_{k=1}^{m}\left(c_{k}+\varepsilon_{k}+\frac{T-t_{k}}{p_{0}} p\left(t_{k}\right)\left(c_{k}^{\prime}+\varepsilon_{k}^{\prime}\right)\right)+\frac{T q}{p_{0}}\right]}<\infty .
$$

Since $\left\|u_{n}\right\|_{\Omega} \rightarrow+\infty$ as $n$ goes to infinity, we arrive to a contradiction. Thus the proof of boundedness of set $E$ is complete. The proof of Theorem 3.3 is complete.

Corollary 3.4. Assume that $\left(H_{4}\right)$ and $\left(H_{5}\right)$ hold. In addition, if one of following conditions holds :

$\left(\mathrm{A}_{3}\right) \lim _{|x| \rightarrow \infty} \frac{\left|I_{k}(x)\right|}{|x|}=0, \lim _{|x| \rightarrow \infty} \frac{\left|J_{k}(x)\right|}{|x|}=0, k=1, \cdots, m$ and $T q<p_{0}$.

$\left(\mathrm{A}_{4}\right)$ There exist constants $c_{k}, c_{k}^{\prime}, b_{k}, b_{k}^{\prime} \in \mathbb{R}$ and $\alpha_{k}, \alpha_{k}^{\prime} \in[0,1)$ such that, for each $x \in R^{n}$,

$$
\left|I_{k}(x)\right| \leq c_{k}|x|^{\alpha_{k}}+b_{k}, \quad\left|J_{k}(x)\right| \leq c_{k}^{\prime}|x|^{\alpha_{k}^{\prime}}+b_{k}^{\prime} \text { and } T q<p_{0} .
$$

Then the problem $(1.1)-(1.3)$ admits at least one solution on $[-\tau, T]$.

Remark 3.2. (1) Let $q(s)=0$ in the problems (1.1)-(1.3), then the Corollary 3.1 and Corollary 3.4 deduce to [6, Theorem 3.2] and [6, Theorem 3.3] respectively.

(2) Impulsive differential equations and inclusions under the conditions $\left(H_{2}\right),\left(A_{2}\right)-$ $\left(A_{4}\right)$ have been widely studied by many authors, see for instance [1], [2], [6], [12]. Obviously, $\left(A_{2}\right)$ and $\left(H_{2}\right)$ are special cases of $\left(H_{6}\right) ;\left(A_{3}\right)$ and $\left(A_{4}\right)$ are special cases of $\left(H_{7}\right)$ and $\left(H_{8}\right)$.

Acknowledgment. The authors are grateful to the reviewers for their valuable comments.

\section{References}

[1] M. Benchohra, J. Henderson and S. K. Ntouyas, On second order multivalued impulsive functional differential inclusions in Banach spaces, Abstract and Applied Analysis, 6(2001), 369-380.

[2] M. Benchohra, J. Henderson and S. K. Ntouyas, On first order impulsive differential inclusions with periodic boundary conditions, Dynam. Contin. Discrete Impuls. Systems, 9(2002), 417-428. 
[3] A. Bressan and G. Colombo, Existence and solutions of maps withdecomposable values, Studia Math., 90(1988), 69-86.

[4] G. L. Cai, The existence of positive solution of impulse neutral delay differential equation, J. Sys. Sci. Math. Scis., 24(1)(2004), 102-109.(In Chinese)

[5] C. Castaing and M. Valadier, Convex analysis and measurable multifunctions, Lecture Notes in Math., Springer-Verlag, Berlin, 580(1977).

[6] Y. K. Chang and W. T. Li, Existence results for second order impulsive differential inclusions, J. Math. Anal. Appl., 301(2005), 477-490.

[7] M. Frigon, Théorèmes d'existence de solutions d'inclusions différentielles, Topological Methods in Differential Equations and Inclusions (edited by A. Granas and M. Frigon), NATO ASI Series C, Kluwer Acad. Publ., 472(1995), 51-87.

[8] V. Lakshmikantham, D. D. Bainov and P. S. Simeonov, heory of Impulsive Differential Equations, World Scientific, Singapore, 1989.

[9] Y. Liu and Z. Li, Schaefer Type Theorem and Periodic Solutions of Evolution Equations, J. Math. Anal. Appl., 316(2006), 237-255.

[10] Y. Liu, J. Wu and Z. Li, Multiple Solutions of Some Impulsive Three-point Boundary Value Problems, DCDIS Proceedings: Impulsive Dynamical Systems and Applications, 3(2005), 579-586.

[11] M. Martelli, A Rothe's type theorem for noncompact acyclic-valued maps, Boll. Un. Mat. Ital., 11(1975), 70-76.

[12] J. J. Nieto, Periodic boundary value problems for first-order impulsive ordinary differential equations, Nonlinear Anal., 51(2002), 1223-1232.

[13] H. Schaefer, Uber die methode der a priori-Schranken, Math. Ann., 129(1955), 415416. 